mountain sickness incidence at high altitude: a prospective randomized trial. Wilderness Environ Med. 2017;28:72-79.

3. McDevitt M, McIntosh SE, Rodway G, Peelay J, Adams DL, Kayser B. Risk determinants of acute mountain sickness in trekkers in the Nepali Himalaya: a 24-year follow-up. Wilderness Environ Med. 2014;25:152-159.

4. Pitman JT, Thapa GB, Stuart NH. Field ultrasound evaluation of central volume status and acute mountain sickness. Wilderness Environ Med. 2015;26:319-326.

5. Lipman GS, Kanaan NC, Phillips C, et al. Study looking at end expiratory pressure for altitude illness decrease (SLEEP-AID). High Alt Med Biol. 2015;16:154-161.

6. Gertsch JH, Corbett B, Holck PS, et al. Altitude sickness in climbers and efficacy of NSAIDs trial (ASCENT): randomized, controlled, trial of ibuprofen versus placebo for prevention of altitude illness. Wilderness Environ Med. 2012; 23:307-315.

7. Gertsch JH, Lipman GS, Holck PS, et al. Prospective, double-blind, randomized, placebo-controlled comparison of acetazolamide versus ibuprofen for prophylaxis against high altitude headache: the headache evaluation at altitude trial (HEAT). Wilderness Environ Med. 2010;2:236-243.

8. Lipman GS, Kanaan NC, Holck PS, Constance BB, Gertsch JH, PAINS Group, Ibuprofen prevents altitude illness: a randomized controlled trial for prevention of altitude illness with non-steroidal anti-inflammatories. Ann Emerg Med. 2012;59:484-490.

\section{In Reply to Drs Lipman and Hackett}

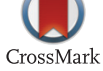

\section{To the Editor:}

We appreciate the discourse from Drs Lipman and Hackett, ${ }^{1}$ value their insights, and welcome the opportunity to discuss our study. ${ }^{2}$ Their main concern is that we have overestimated the expected incidence of acute mountain sickness (AMS). We echo this concern and wish we could accurately know the true incidence of AMS at our study site. We attempted to estimate it as accurately as possible using available data. The studies they cited are not ideal for direct comparisons because they used different locations, elevations, ascent rates, methodologies, and inclusion/exclusion criteria than those in our study. Because of the site-specific variability of AMS incidence, it is important to compare only those studies that are nearly identical.

They state that there has been an "almost a $50 \%$ decline in AMS over the past 18 years, to $22 \%$ at the Himalayan Rescue Association clinic in Manang." The studies conducted at $3519 \mathrm{~m}$ in Manang, Nepal, had a completely different trekking ascent profile and location. Our study was performed $\sim 300 \mathrm{~km}$ away at a $\sim 1000 \mathrm{~m}$ higher elevation, in Pheriche $(4371 \mathrm{~m})$ and Dingboche (4410 m), to Lobuche (4940 m). Although we agree with their inference that a lower incidence poses challenges tin studying altitude illness in Manang, Manang was not our study site, nor was our methodology similar to that in the study they cited.

The SLEEP-AID study ${ }^{3}$ cited did indeed originate in Pheriche and Dingboche. Unfortunately, this study was also not comparable with ours because AMS incidence was studied at different altitudes. The SLEEP-AID study was an overnight study while participants were staying in Pheriche and Dingboche. Our study and those we relied on for our power analysis followed participants for at least 2 days as they ascended over $500 \mathrm{~m}$ higher to Lobuje, where AMS incidence was then calculated. One would expect to find lower AMS incidence among subjects at a lower altitude for a shorter duration than in subjects trekking to a higher altitude followed over several days.

The authors argue that without a placebo arm one does not "know if these medications reduced the incidence of AMS at all." We agree, but to identify or quantify the efficacy of these treatments relative to placebo was never the intent of our study. Rather, our study purpose was to compare the efficacy of 2 treatments (ibuprofen and acetaminophen) relative to each other in modifying AMS incidence. Questions examining the efficacy of these treatments relative to placebo have been addressed in numerous previous studies elsewhere. We did note that "combined group incidence of AMS was 19.1\%, which is notably lower than the $33 \%$ averaged historical incidence" and surmised that this apparent decrease in AMS incidence in both treatment groups relative to historical placebo levels likely resulted in part due to both treatments reducing AMS. This hypothesized explanation was based on prior studies showing ibuprofen's efficacy at AMS prevention. ${ }^{4}$ If indeed historical overall reductions of AMS incidence in trekkers at our study site are taking place, that too could provide an additional explanation for at least some of the reduced AMS incidence we observed. That said, we examined whether ibuprofen and acetaminophen differed in the resulting incidence of AMS. We found little evidence to suggest that they do and concluded that "acetaminophen performs similarly to ibuprofen in the prevention of AMS in partially acclimatized subjects."

We concluded that there was no strong evidence of a difference. Our reported results specify the strength of that conclusion based on sample size. We calculated that the difference in AMS incidence between the two treatments was $7 \%$, but concluded that in the population overall, the true difference might be anywhere between $-4 \%$ and $+16 \%$. The uncertainly of our conclusion illustrates a limitation of sample size, but it does provide some likely bounds regarding what differences are likely, should any exist, between the medications. Recruiting a third "placebo" arm to the study would have required either fewer 
subjects in our treatment arms or necessitated extending the trial over multiple trekking seasons. Possible declines in AMS incidence are far from conclusive. Larger sample sizes of Nepal trekkers (including multiseason recruitment) may be necessary for more precise estimates. Regardless, neither of these potential concerns invalidates our conclusions or indicates that we used an inappropriate approach to examine differences between ibuprofen and acetaminophen for prevention of AMS.

Lipman and Hackett's estimation of our power calculations for our test of equivalency needs slight correction. We anticipated an incidence of about 14\% AMS in our treatment groups, but we wanted to be able to detect a difference as small as $11 \%$ between the 2 treatments, a difference that might be clinically relevant. For $80 \%$ power and $\alpha=0.05$, we estimated we would need at most 125 individuals in each arm. Although we recruited more than that number of subjects, exclusions and a slightly higher than expected incidence of AMS resulted in a less precise estimate of the magnitude of difference between treatment arms.

Finally, we would also like to make a small correction: Our manuscript stated an AMS incidence of 33\% in our power calculation, not $32 \%$ as was cited. For the sake of openness and clarity, it must be stated that we conducted our power analysis before conducting the study, based on multiple studies ${ }^{5-8}$ with the same location, elevation, ascent profiles, recruitment methodologies, and time course. To calculate the historical AMS incidence, we combined the results of these studies by dividing the number of subjects who developed AMS in the placebo arms by the total number of subjects in the placebo arms (omitting subjects who received interventions), or $44+16+40+20=120$ divided by $109+53+119+81=$ 362. The overall incidence of AMS in subjects taking placebo in these studies was $120 / 362$, or $33 \%$.

Nicholas C. Kanaan, MD

Alicia L. Peterson, MD

Division of Emergency Medicine, University of Utah

Matiram Pun, MD

Ghan B. Thapa, MD

Aditya Tiwari, MD

Bikash Basyal, MD

The Institute of Medicine, Maharajgunj, Kathmandu Nepal

Peter S. Holck, PhD

The Department of Public Health, University of Hawaii

Jennifer Starling, MD

The Department of Emergency Medicine, Denver Health
Thomas F. Freeman, MD

The Department of Emergency Medicine

Augusta University

Jessica R. Gehner, MD

The Emergency Medicine Residency

Virginia Tech Carilion

Linda Keyes, MD

The Department of Emergency Medicine University of Colorado, Aurora, Colorado

Dana R. Levin, MD

The Department of Aerospace Medicine University of Texas Medical Branch

Catherine J. O'Leary, MD

The Department of Emergency Medicine Thomas Jefferson University Hospital

Katherine E. Stuart, MD

The Department of Emergency Medicine Queen's University, Kingston ON, Canada

Jared L. Velgersdyk, MD The Department of Internal Medicine University of North Dakota

Ken Zafren, MD

The Department of Emergency Medicine Stanford University Medical Center

Stanford, $C A$

and the Himalayan Rescue Association

Buddha Basnyat, MD

The Oxford University Clinical Research Unit

Kathmandu, Nepal

and the Himalayan Rescue Association and the Centre for Tropical Medicine and Global Health University of Oxford, UK

\section{References}

1. Lipman GS, Hackett P. In response to ibuprofen vs acetaminophen in AMS prevention by Kanaan et al. Wilderness Environ Med. 2017;28:383-385.

2. Kanann NC, Peterson AL, Pun M, et al. Prophylactic acetaminophen or ibuprofen result in equivalent acute mountain sickness incidence at high altitude: a prospective randomized trial. Wilderness Environ Med. 2017;28:72-79.

3. Lipman GS, Kanaan NC, Phillips C, et al. Study looking at end expiratory pressure for altitude illness decrease (SLEEP-AID). High Alt Med Biol. 2015;16:154-161. 
4. Lipman GS, Kanaan NC, Holck PS, Constance BB, Gertsch JH, PAINS Group, Ibuprofen prevents altitude illness: a randomized controlled trial for prevention of altitude illness with nonsteroidal anti-inflammatories. Ann Emerg Med. 2012;59:484-490.

5. Gertsch JH, Corbett B, Holck PS, et al. Altitude Sickness in Climbers and Efficacy of NSAIDs Trial (ASCENT): randomized, controlled trial of ibuprofen versus placebo for prevention of altitude illness. Wilderness Environ Med. 2012;23:307-315.

6. Gertsch JH, Lipman GS, Holck PS, et al. Prospective, double-blind, randomized, placebo-controlled comparison of acetazolamide versus ibuprofen for prophylaxis against high altitude headache: the Headache Evaluation at Altitude Trial (HEAT). Wilderness Environ Med. 2010;21:236-243.

7. Gertsch JH, Basnyat B, Johnson EW, et al. Randomised, double blind, placebo controlled comparison of ginkgo biloba and acetazolamide for prevention of acute mountain sickness among Himalayan trekkers: the prevention of high altitude illness trial (PHAIT). BMJ. 2004;328: 797-799.

8. Basnyat B, Gertsch JH, Johnson EW, Castro-Marine E, Inoue Y, Yeh C. Efficacy of low-dose acetazolamide (125 mg BID) for the prophylaxis of acute mountain sickness: a prospective, double-blind, randomized, placebo-controlled trial. High Alt Med Biol. 2003;4:45-52. 